Western University

Scholarship@Western

$1-2018$

\title{
A Mass-Producible and Versatile Sensing System: Localized Surface Plasmon Resonance Excited by Individual Waveguide Modes
}

Zhutian Ding

Western University

James M. Stubbs

Western University

Danielle McRae

Western University

Johanna M. Blacquiere

Western University

François Lagugné-Labarthet

Western University

See next page for additional authors

Follow this and additional works at: https://ir.lib.uwo.ca/physicspub

Part of the Astrophysics and Astronomy Commons, and the Physics Commons

Citation of this paper:

Ding, Zhutian; Stubbs, James M.; McRae, Danielle; Blacquiere, Johanna M.; Lagugné-Labarthet, François; and Mittler, Silvia, "A MassProducible and Versatile Sensing System: Localized Surface Plasmon Resonance Excited by Individual Waveguide Modes" (2018).

Physics and Astronomy Publications. 36.

https://ir.lib.uwo.ca/physicspub/36 
Authors

Zhutian Ding, James M. Stubbs, Danielle McRae, Johanna M. Blacquiere, François Lagugné-Labarthet, and Silvia Mittler 
ACS Sensors 3 (2018) 334-341

\title{
A Mass-Producible and Versatile Sensing System: Localized Surface Plasmon Resonance Excited by Individual Waveguide Modes
}

\author{
Zhutian Dinga,b, James M. Stubbs ${ }^{\mathrm{b}}$, Danielle McRae ${ }^{\mathrm{b}}$, Johanna M. Blacquiere ${ }^{\mathrm{b}}$, François Lagugné- \\ Labarthet $^{\mathrm{b}}$, Silvia Mittler ${ }^{*, \mathrm{a}, \mathrm{b}}$ \\ ${ }^{a}$ Department of Physics and Astronomy, The University of Western Ontario, 1151 Richmond Street, London, On- \\ tario, Canada N6A $3 \mathrm{~K}_{7}$ \\ ${ }^{\mathrm{b}}$ Department of Chemistry, The University of Western Ontario, 1151 Richmond Street, London, Ontario, Canada \\ $\mathrm{N} 6 \mathrm{~A}{ }_{5} \mathrm{~B} 7$
}

Keywords: Gold nanoparticles, localized surface plasmon resonance, optical sensor, waveguide, biosensing

\begin{abstract}
A plasmonic sensing system that allows the excitation of localized surface plasmon resonance (LSPR) by individual waveguide modes is presented conceptually and experimentally. Any change in the local environment of the gold nanoparticles (AuNPs) alters the degree of coupling between LSPR and a polymer slab waveguide, which then modulates the transmission-output signal. In comparison to conventional LSPR sensors, this system is less susceptible to optical noise and positional variation of signals. Moreover, it enables more freedom in the exploitation of plasmonic hot spots with both transverse electric (TE) and transverse magnetic (TM) modes. Through real-time measurement, it is demonstrated that the current sensing system is more sensitive than comparable optical fiber plasmonic sensors. The highest normalized bulk sensitivity $\left(7.744 \mathrm{RIU}^{-1}\right)$ is found in the $\mathrm{TM}_{1}$ mode. Biosensing with the biotin-streptavidin system shows that the detection limit is on the order of $10^{-14} \mathrm{M}$ of streptavidin. With further optimization, this sensing system can easily be mass-produced and incorporated into high throughput screening devices, detecting a variety of chemical and biological analytes via immobilization of the appropriate recognition sites.
\end{abstract}

Localized surface plasmon resonance (LSPR) sensors spark intensive research due to their potential applications in disease diagnosis, drug discovery, proteomics, and environmental detection. ${ }^{1}$ Gold nanoparticles (AuNPs) exhibit LSPR when the incident photon frequency matches the oscillation frequency of conduction electrons. ${ }^{1}$ The resonance results in a strong UV-Vis absorption band whose frequency is highly dependent on the size, shape, distribution, dielectric properties, and local environment of the AuNPs. ${ }^{2}$ This frequency sensitivity towards local refractive index enables the transduction of binding events on the AuNPs into optical signals: changes in LSPR resonance wavelength, extinction, or both. ${ }^{1,3}$

The incorporation of a waveguide into LSPR sensor system for absorption-based measurement overcomes four limitations of a conventional plasmonic sensor that works in the direct transmission mode. First, the traditional configurations are susceptible to optical noise introduced by irrelevant molecules that scatter or absorb as light propogates. ${ }^{4-5}$ Waveguides, on the other hand, guide light with low loss and are relatively immune to outside interferences. Second, positional variation of signals resulting from the nonuniform and polydisperse nature of the nanoparticle population compromises the reproducibility of sensor behavior. In contrast, waveguide sensors detect the integral behavior of all the nanoparticles inside a sensing area, leading to a higher reproducibility. Third, due to the spectroscopic nature of conventional sensors, bulky prims and moving optical components present engineering challenges for high throughput screening applications. ${ }^{6}$ On the contrary, a system of slab waveguide combined with a monochromatic light source is structurally robust and highly miniaturizable. ${ }^{7}$ Lastly, transmission-based sensors limit the exploitation of nonspherical plasmonic features that are sensitive to polarized light because the electric field of incident light cannot oscillate in its direction of propagation. The use of a waveguide liberates this limitation. 
Herein, conceptually and experimentally, a mass-producible and versatile plasmonic sensing system that allows the excitation of LSPR by individual waveguide modes is presented. This system can be adapted by research groups working on plasmonic nanoparticles of various shapes and sizes, and is able to detect a variety of chemical and biological analytes by immobilizing the appropriate recognition sites on the nanoparticles. To demonstrate the advantages of the system, the mode-specific bulk sensitivity of pancake shaped AuNPs immobilized onto the waveguide surface via organometallic chemical vapor deposition (OMCVD) is studied. First, the quality of the slab waveguide before and after gold deposition is characterized via $\mathrm{m}$-line spectroscopy. Through real-time measurement, it is demonstrated that the current sensing system is more sensitive than comparable optical fiber plasmonic sensors ${ }^{8-9}$. Furthermore, optimization strategies in the context of the sensitivity study are discussed.

\section{EXPERIMENTAL SECTION}

Materials. Chloro(trimethylphosphine)gold(I) (99\%), methyl lithium (1.6 $\mathrm{M}$ in diethyl ether), lithium aluminum hydride (95\%), magnesium sulfate, chlorobenzene, trichloro(octadecyl)silane (OTS), 11-mercapto-1-undecanol (97\%; referred to as alkane thiol hereafter), and silicon sheets were acquired from Sigma Aldrich (Ontario, Canada). Sulfuric acid (98\%), hydrogen peroxide (30\%), ethanol, glycerol, and toluene were obtained from Caledon Laboratories Ltd. (Ontario, Canada). Polystyrene granules were purchased from Goodfellow Cambridge Ltd. (Huntingdon, England). Monothiolalkane PEG-biotin was purchased from SensoPath Technologies (Montana, USA). Streptavidin was obtained from Rocklan Immunochemicals Inc. (Pennsylvania, USA). Diethyl ether was distilled from lithium aluminum hydride and degassed before use. Water used during this study was deionized, obtained from Millipore cartridgesAll other materials were used without further purification. Fused silica substrates with coupling grating $(2.5 \mathrm{~cm} \times 5 \mathrm{~cm}, 1 \mathrm{~cm}$ between the top and the grating edge) were fabricated at the RheinMain University of Applied Sciences Wiesbaden, Rüsselsheim, Germany. OMCVD was carried out in a custom-made glass chamber.

OMCVD. Methyl(trimethylphosphine)gold(I) was synthesized as previously described in the literature. ${ }^{10}$ AuNPs were deposited as previously described with modifications. ${ }^{10}$ Information regarding the instrumentation (spin coater and UV ozone generator) is documented in the same paper..$^{10}$ Fused silica substrates carrying a coupling grating were used as waveguide substrates. The spin coating solution was prepared by dissolving $1.5 \mathrm{~g}$ of polystyrene in $13 \mathrm{~mL}$ of chlorobenzene. The spin coating speed for the polystyrene waveguide was $2200 \mathrm{rpm}$. The baking process after spin coating was omitted. A piece of silicon sheet, the same size as the waveguide, with a circle carved out $(1.6 \mathrm{~cm}$ in diameter, $1.5 \mathrm{~cm}$ away from the top), was placed on top of the polystyrene waveguide during UV ozone treatment. Additionally, o. $6 \mathrm{~mL}$ of hydrogen peroxide was added into the UV ozone chamber to enhance -OH group creation on the polystyrene waveguide. OMCVD was performed at 65.5 ${ }^{\circ} \mathrm{C}$ for 11 min $30 \mathrm{~s}$ under $13.5 \mathrm{~Pa}$.

Optical setup and UV-Vis spectroscopy. Modified from the literature, ${ }^{11}$ the optical setup of m-line spectroscopy and bulk sensing is essentially the same. Light emitted from a red HeNe laser $(632.8 \mathrm{~nm}, 10 \mathrm{~mW}$; JDS Uniphase, USA) passes through an optical chopper (Signal Recovery 197, Ametek Inc. USA), a linear polarizer (Newport Corporation, USA), and an iris (Thorlabs ID8, USA) before reaching the edge of the coupling grating. Waveguide transmission is measured with a photodiode pressed against its end face. The photodiode signal is modulated by a lock-in amplifier (Signal Recovery 7265 DSP) for noise reduction. A sample holder, clamping the waveguide and a teflon flow cell ( $1 \mathrm{~mL}$ ) in place, is situated on a three-axis mount (Owis, Germany), which is controlled by a goniometer (Huber, Model 414, Germany). Both the goniometer and the lock-in amplifier are connected to a computer. All optical experiments were carried out at $20{ }^{\circ} \mathrm{C}$. The $\mathrm{m}$-line spectrometer was scanned in increments of $0.025^{\circ}$. The angle of incidence is defined as the angle between the incident light and the surface normal (y-axis of Fig.1). Coupling angles for individual modes are found at angles with the highest intensities at end face. A software package (MPI for Polymer Research, Mainz, Germany) is used to control the m-line setup. It has two operation modes: a) an m-line mode where the waveguide transmission is measured as a function of incident angle, and b) a kinetic mode where the signal at the end face is measured over time at a fixed coupling angle position. UV-Vis spectroscopy in transmission (perpendicular to the propagation direction of waveguide modes, along y-direction of Fig.1) was performed with a Lambda 850 UV-Vis spectrometer (Perkin Elmer, USA) to gain information about the spectral response of the AuNPs.

Bulk sensing. Bulk sensing was carried out in the kinetic mode at the coupling angles obtained from $\mathrm{m}$-line spectroscopy. Starting with water, $2 \%, 4 \%, 5 \%, 7 \%, 9 \%$, $11 \%$, and $13 \%$ of glycerol solutions (by weight) were introduced into the flow cell. The measurement was conducted with $\mathrm{TE}_{\mathrm{o}}, \mathrm{TE}_{1}, \mathrm{TM}_{\mathrm{o}}$, and $\mathrm{TM}_{1}$ waveguide modes. A data point was recorded every $5 \mathrm{~s}$.

\section{RESULTS AND DISCUSSION}

Sensing mechanism and theoretical sensitivity. The key elements of the sensing system are illustrated in Fig. 1. Light propagating within the waveguide assumes either a transverse electric (TE, s-polarized light) or a transverse magnetic (TM, p-polarized light) mode. Coupling between the evanescent fields of a waveguide mode and an LSPR mode is contingent upon resonance conditions of the AuNPs being satisfied. Because of this strong coupling, light intensity within the waveguide diminishes. Any increase in local refractive index of the AuNPs leads to a redshift of their extinction peak. Consequently, the coupling is attenuated, and the waveguide transmission signal rebounds. Since scattering behavior of the AuNPs does not contribute substantially to waveguide transmission, we 
will simplify the situation by considering absorption spectra only.

The bulk sensitivity of the system is defined as the change of signal output divided by the change in refractive index, normalized by the signal of water (or any blank defined by the user). All the liquid media mentioned in this paper are assumed to be free of charge, homogeneous, and isotropic. Consider a hypothetical absorption spectrum of AuNPs in water described by function $A(\lambda)$. When a liquid of higher refractive index is introduced, the absorption curve exhibits a redshift of $\Delta \lambda \mathrm{nm}$ as indicated by the horizontal arrow in Fig. 2. If the increase in refractive index is small enough, we can assume that the resulting absorption spectrum is a redshifted version of the original one. The definition of "small" is specific to the size, shape, distribution, and dielectric environment of AuNPs. Thus, the shifted spectrum can be described by function $\mathrm{A}(\lambda-\Delta \lambda)$. We can then approximate $\mathrm{A}(\lambda-\Delta \lambda)$ in terms of $\mathrm{A}(\lambda)$ using Taylor expansion, where $\mathrm{D}^{\prime}$ is the first derivative of absorption with respect to wavelength, $\mathrm{D}^{\prime}$ is the second, and so on.

$$
A(\lambda-\Delta \lambda)=A(\lambda)-\Delta \lambda D^{\prime}+\frac{(\Delta \lambda)^{2}}{2} D^{\prime \prime}-\frac{(\Delta \lambda)^{3}}{6} D^{\prime \prime \prime}+\cdots
$$

Since the coupling conditions are unaltered throughout the experiment, the sensing area has a constant feed of input intensity. The transmission signal increase with respect to water $(\Delta \mathrm{I})$ upon injection of another liquid with a higher refractive index $(\Delta \mathrm{n})$ is simply the negative of the difference between $A(\lambda)$ and $A(\lambda-\Delta \lambda)$ at the observation wavelength as marked by the vertical arrow in Fig. 2.

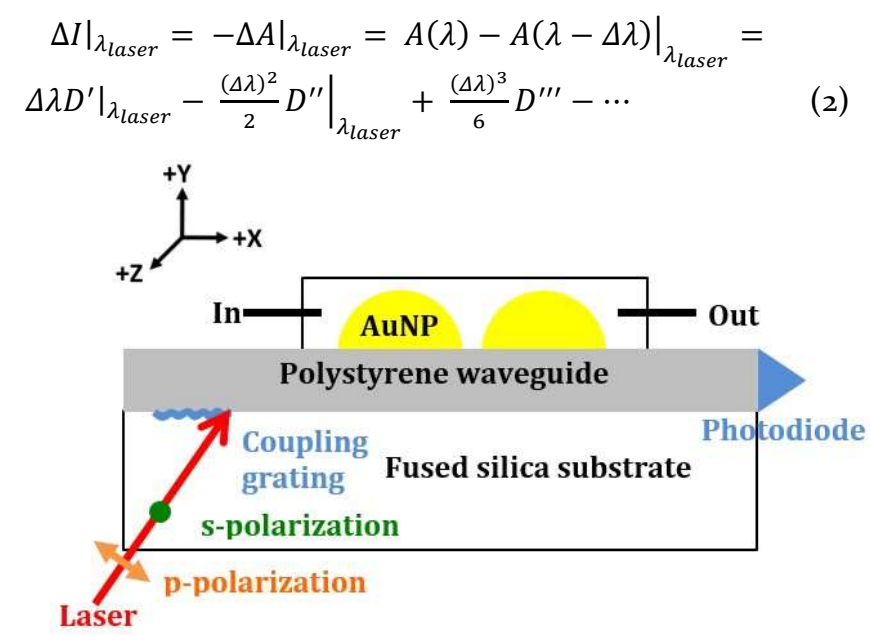

Fig. 1. Schematic of the waveguide sensor. A substrate (white) carrying a coupling grating (blue) supports a polystyrene waveguide (grey) with AuNPs (yellow) covalently bound on top. A flow cell is fixed to cover a circular sensing area where AuNPs are deposited. Mode-selective coupling is conducted outside the flow cell to ensure constant coupling conditions. The intensity of a transmitted waveguide mode is detected by a photodiode (blue triangle) at its end-face. The electrical field oscillates in the xy-plane for p-polarization (orange double arrow), and along the z-axis for s-polarization (green dot).
When the redshift is small enough, we can approximate the signal increase with only the first term. Note that the first derivative of absorbance with respect to wavelength $\left(\mathrm{D}^{\prime}\right)$ evaluated at the observation wavelength is a constant for a given spectrum. The unit of $D^{\prime}$ is a.u./nm. Because the LSPR peak wavelength is approximately linear with respect to refractive index $\left(n_{i}\right)$ within a certain range, ${ }^{2}$ we can decompose $\Delta \lambda$ using two proportionality constants, $\mathrm{S}_{\text {wavelength }}$ and R. $S_{\text {wavelength }}$ is the sensitivity of LSPR peak wavelength in $\mathrm{nm} / \mathrm{RIU}$. $\mathrm{R}$ is the response constant of the photodiode (or other detectors) in mV/a.u. According to the definition earlier, the theoretical bulk sensitivity of this absorptionbased system, $\mathrm{S}_{\mathrm{abs}}$, within a linear range in $\mathrm{RIU}^{-1}$ is:

$$
S_{\text {abs }}=\frac{I_{\text {liquid }}-I_{\text {water }}}{\Delta n}=D^{\prime} S_{\text {wavelengt }} R
$$

Conventionally, the bulk sensitivity of an LSPR sensor is defined as the rate of wavelength shift versus refractive index change, normalized by the full width of half maximum (FWHM) of the resonance peak $\left(\mathrm{S}_{\text {wavelength }} / \mathrm{FWHM}\right.$ in RIU$\left.{ }^{1}\right) .{ }^{12} \mathrm{~A}$ narrow ensemble peak entails rigorous control in AuNP's synthetic techniques, which is necessary to improve the wavelength sensitivity. On the other hand, the sensitivity of this proposed system is independent of FWHM and is amplified by the slope term ( $\left.\mathrm{D}^{\prime}\right)$.

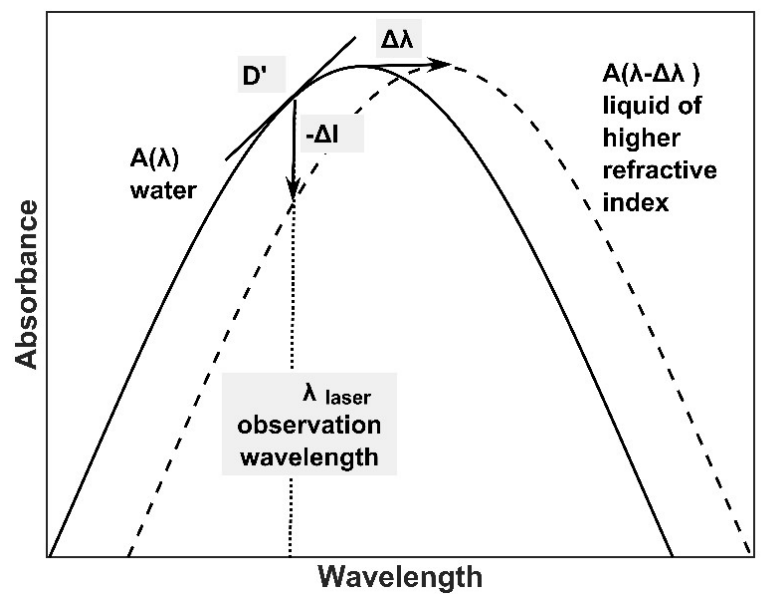

Fig. 2. A hypothetical redshift of an absorption spectrum of AuNPs induced by a change in bulk refractive index, $\Delta \mathrm{n} . \mathrm{A}(\lambda)$ : spectrum in water, solid curve; $A(\lambda-\Delta \lambda)$ : spectrum in a liquid of higher refractive index, dashed curve; $\Delta \mathrm{I}$ at the observation wavelength, vertical arrow; $\Delta \lambda$, horizontal arrow.

Characterization of AuNPs. Polystyrene, like most polymers, is inert towards surface functionalization unless treated with harsh chemicals. Such treatments undermine the integrity and high transparency of polymer waveguides. In comparison, UV-activated ozone is relatively mild and suitable for mass production. Reactive gaseous species generated by the photolysis of ozone react with the polystyrene surface, introducing surface hydroxyl groups through the combination of hydroxyl and carbon radicals. ${ }^{13}$ These hydroxyl groups are nucleation sites for $\mathrm{Au}(0)$ nanoparticles that are formed through successive bimolecular 
reductive elimination reactions between monoalkyl Au(I) species. ${ }^{14-15}$

However, UV ozone treatment alone did not guarantee a successful gold deposition every time. There were instances where methyl(trimethylphosphine)gold(I) simply re-condensed inside the reaction chamber and no AuNPs were observed at all. The addition of hydrogen peroxide during UV ozone treatment significantly increases the success rate of gold deposition to almost 100\%. This might result from an increased surface density of hydroxyl groups, which facilitates the transfer of methyl group from one methyl gold to an adjacent gold center. ${ }^{16}$

The LSPR peak wavelength shifts by $10 \mathrm{~nm}$ as the bulk refractive index increases from 1.333 to 1.361 (Fig. 3A). The resonance wavelength is significantly redshifted in comparison to colloidal AuNPs of similar size because of the substrate effect ${ }^{17}$ and possibly the interaction between gold and hydroxyl groups. Since the shape of the absorption curve does not change significantly, we expect the linear range of sensor performance is approximately between 1.333 and 1.361. The shoulder around $575 \mathrm{~nm}$ might be the quadrupole peak that is exposed after the dipolar peak is redshifted. ${ }^{18}$ The second peak in the $700 \mathrm{~nm}$ regime result from the plasmonic coupling of adjacent nanoparticles. ${ }^{19-20}$

The diameter distribution (Fig. $3 \mathrm{~B}$ ) of the AuNPs was measured with a scanning electron microscope (SEM; Leo 1540 XB, Carl Zeiss, Oberkochen, Germany). $15 \mathrm{~nm}$ of aluminum was sputtered on the polystyrene surface before the AuNPs were studied under $20 \mathrm{kV}$ beam in back-scatter mode (Fig. ${ }_{3} \mathrm{C}$ ). The zoomed inset reveals the presence of some clusters, which could result either from melting of the polymer substrate during electron bombardment or from the aluminum deposition process. The mean diameter of the AuNPs was found to be $19 \pm 1 \mathrm{~nm}$.

The topography of the AuNPs was analyzed with an atomic force microscope (AFM; NanoWizard II, JPK Instruments Inc.) in non-contact mode with $512 \times 512$ points per image. Commercial silicon tips (NCL-50, NanoWorld Inc.), with resonance frequency of $190 \mathrm{kHz}$ and force constant of $48 \mathrm{~N} / \mathrm{m}$, were used in the measurements. The images were processed by taking cross-sections of particles, then determining the height. The average height of 50 particles investigated was found to be $7 \pm 3 \mathrm{~nm}$. We therefore deal with pancake shaped AuNPs with an aspect ratio (height/lateral) of 7:19. This finding is consistent with previous studies on OMCVD grown AuNPs with the same precursor. Although a different substrate functionalizing selfassembled monolayer (-SH instead of $-\mathrm{OH}$ ) was used, the general outline of those AuNPs was found to be pancake shaped as well with aspect ratios (height/lateral) of 1/1.5, $1 / 10$ and $1 / 1.4 \cdot{ }^{19}$

Waveguide mode profiles before and after gold deposition. M-line spectroscopy scans angle of incidence as a function of waveguide transmission for both polarizations, the maxima of which correspond to the coupling angles for individual modes. These data are typically used to calculate the thickness and refractive index $\left(n \propto \sqrt{\varepsilon^{\prime}}, \varepsilon^{\prime}\right.$ is the real part of dielectric function) of a waveguide, if the optical data of its substrate and coupling grating are known. In a classic $\mathrm{m}$-line, the area of mode coupling is identical to the rest of the waveguide. The peak width delivers information on the imaginary part $\left(\varepsilon^{\prime \prime}\right)$ of the dielectric function $(\varepsilon=$ $\left.\varepsilon^{\prime}+\mathrm{i} \varepsilon^{\prime \prime}\right)$, thus describes the absorption and scattering features of the waveguide. In our case, characterization of the waveguide after gold deposition (Fig. 4, black line) differs
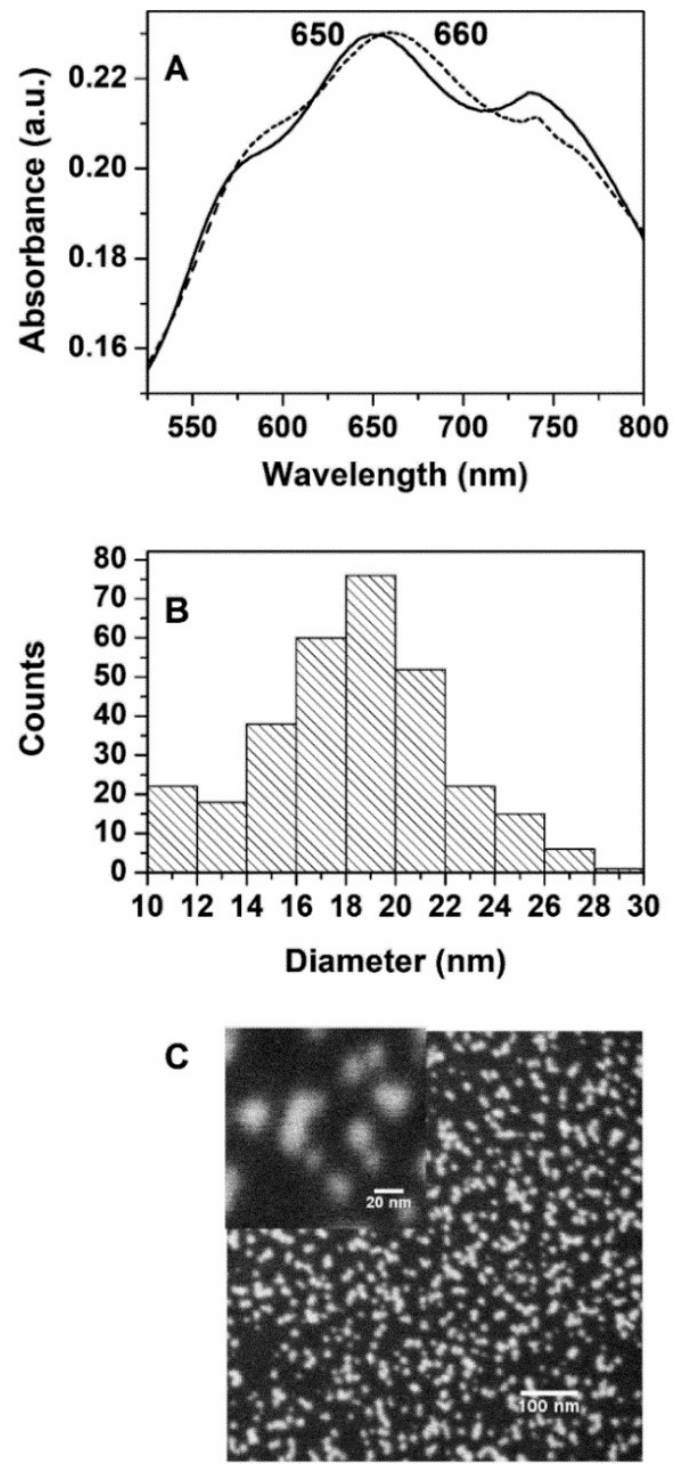

Fig. 3. (A) UV-Vis absorption spectra of AuNPs in water (solid) and ethanol (dashed) measured in transmission (along y-axis of Fig. 1). (B) Histogram of particle diameters from SEM image. (C) SEM image of OMCVD AuNPs on polystyrene: large scale bar $100 \mathrm{~nm}$, small scale bar $20 \mathrm{~nm}$. These images are of low quality because a short integration time was used due to the unstableness of the polystyrene substrate. High-resolution images require a relatively long integration time, which damages the polymer substrate due to electron bombardment and local heating.

from the classic operation in that coupling is performed outside the area with AuNPs and that waveguide transmission is collected after certain degree of energy transfer to AuNPs. As a result, the peak intensities decrease and the 
peak widths increase in comparison to the bare waveguide (Fig. 4, grey spectra). Simulations with Fresnel equations for waveguide modes reveal increased peak widths and decreased peak heights with increasing absorpt ion in the system. Therefore three related parameters are considered: coupling angle, peak intensity, and peak width. The coupling angle is directly related to the effective refractive index of a guided mode under its coupling conditions. ${ }^{21}$

Table 1. Parameters that quantify the extent of energy transfer from the waveguide to the AuNPs

$\begin{array}{llllll} & & \mathrm{TM}_{\mathrm{o}} & \mathrm{TM}_{1} & \mathrm{TE}_{\mathrm{o}} & \mathrm{TE}_{1} \\ \text { Bare waveguide } & \text { IOWA }(\% / \mathrm{mV}) & 0.053 & 0.013 & 0.018 & 0.0039 \\ \text { After gold deposition } & \text { IOWA }(\% / \mathrm{mV}) & 0.072 & 0.027 & 0.073 & 0.026 \\ & \text { IONA } & 0.36 & 1.0 & 3.0 & 5.8\end{array}$

The errors associated with these parameters are detailed in Table S1.

Because the coupling conditions of individual modes remain unaltered before and after gold deposition, their coupling angles, or as depicted in Fig. 4, their peak positions remain unchanged given the uncertainty in angle measurement is $\pm 0.025^{\circ}$.

To quantify the extent of energy transfer from the waveguide to the AuNPs, we define two normalized parameters: the index of waveguide absorption (IOWA) and the index of nanoparticle absorption (IONA). IOWA $=\mathrm{FWHM} / \mathrm{I}_{\max }$ in $(\% \mathrm{mV})$. IONA $=\left(\mathrm{IOWA}_{\text {AuNP }}-\mathrm{IOWA}_{\text {bare }}\right) / \mathrm{IOWA}_{\text {bare, }}$, which describes the contribution of the AuNPs to coupling peaks measured at the end face. The m-line peaks were fitted with a Gaussian model using Origin Pro 8, from which values of FWHM were extracted. An example is shown in Fig.S1 of the Supporting Information. Table 1 summarizes the two parameters for all four guided modes. A more exhaustive table on the parameters of the coupling peaks can be found in Table S1, together with their experimental errors. The peak intensity, $I_{\max }$, is the value displayed on the lock-in amplifier when the waveguide is turned to the coupling angle of a certain mode.

IONA signifies the normalized proportion of guided light absorbed by AuNPs. The difference between the two polarizations is caused by the distinct absorption behavior when pancake shaped AuNPs are illuminated along different axes (see Fig. 1 for the coordinate system), which ultimately stems from their unsymmetrical shape and dielectric environment. TE modes probe along the $\mathrm{z}$-axis or the long axes of the pancake shaped AuNPs, whereas TM modes probe mostly in y-direction or along their short axes (a small $\mathrm{x}$-component is ignored for simplicity). ${ }^{22}$ Because the long axis contains more material than the short axis, the particles illuminated by TE modes absorb more light than those excited by TM modes as confirmed by the IONA values in Table 1 . Note that lower IONA values of TM modes do not directly translate to less amount of guided light being absorbed because they are normalized values. However, due to the facts that TM modes carry less light before gold deposition and that less proportion of light is absorbed after gold deposition, we can draw the above connections between AuNP absorption and IONA values of two polarizations.
Within both polarization directions, the IONA value for the first mode $\left(\mathrm{TM}_{1}\right.$ and $\left.\mathrm{TE}_{1}\right)$ is higher than the zeroth mode $\left(\mathrm{TM}_{\mathrm{o}}\right.$ and $\left.\mathrm{TE}_{\mathrm{o}}\right)$. Here, the difference in absorption cross section originates from the evanescent fields of different waveguide modes: with increasing mode number,

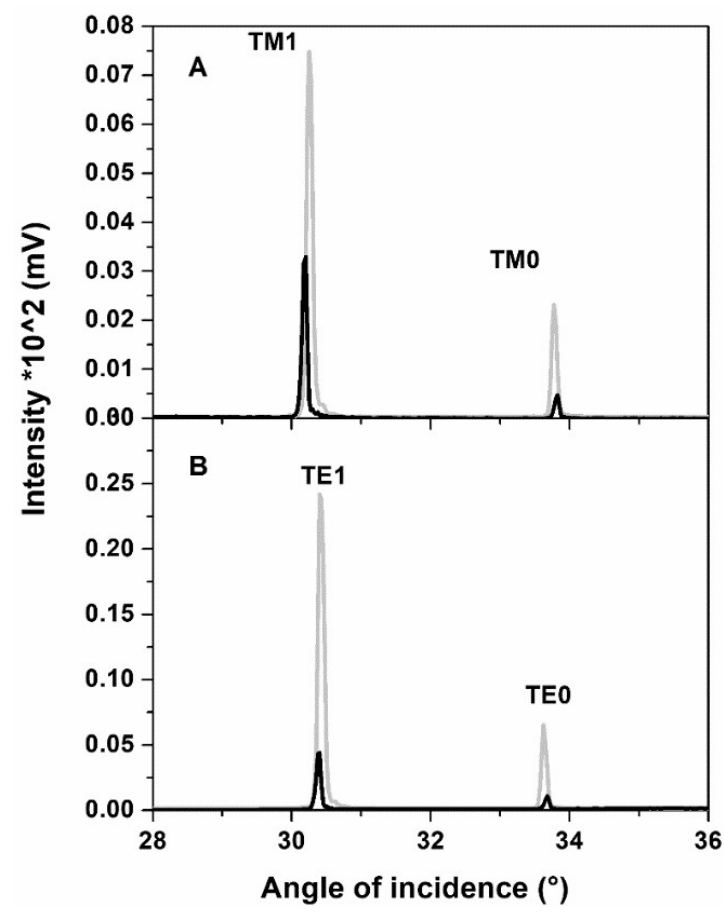

Fig. 4. Baseline corrected m-line spectra for (A) p-polarization and (B) s-polarization. Bare waveguide, grey; waveguide after gold deposition, black. Please note the difference in the intensity scale for s- and p-polarization.

the integrated mode intensity increases. ${ }^{23}$ Consequently, within a chosen polarization, LSPR excited by the higher mode generates a more intense plasmonic evanescent field, which leads to a superior sensitivity as we will see in the next section. However, bulk sensitivities for different polarizations cannot be extrapolated from m-line data because higher absorption does not equate to higher sensitivity (rate of change of absorption with respect to refractive index) when AuNPs are illuminated along different axes. In conclusion, we find from the m-line investigation 
that coupling condition remains unchanged before and after gold deposition. The argument of a constant input in the theoretical derivation is thus valid. Sharp and defined $\mathrm{m}$-line peaks before and after gold deposition translate to a high-quality waveguide, which ensures its reliable sensing behavior.

Mode-specific bulk sensitivity. The real-time waveguide transmission of the $\mathrm{TE}_{1}$ mode (at its coupling angle) is depicted in Fig. 5A. See Fig. S2 (Supporting Information) for the kinetic measurements of the other three modes $\left(T E_{o}, \mathrm{TM}_{\mathrm{o}}\right.$ and $\left.\mathrm{TM}_{1}\right)$. The mode-specific average waveguide transmissions are tabulated in Table $\mathrm{S}_{2}$. The slopes of the linear regression lines (Fig. ${ }_{5} \mathrm{~B}$ ) represent the normalized bulk sensitivities for individual modes in RIU ${ }^{-1}$. Their numerical values are 2.654 for $\mathrm{TM}_{0}, 7.744$ for $\mathrm{TM}_{1}, 1.500$ for $\mathrm{TE}_{\mathrm{o}}$, and 2.909 for $\mathrm{TE}_{1}$. Note that the normalized sensitivity for each mode is calculated using the values in Table S2 divided by the transmission signal for water in that mode.

Although liquid media can absorb or scatter photons while in the evanescent field of the waveguide, they barely contribute to the change in waveguide transmission as the chosen liquids are close to $100 \%$ transparent at the observation wavelength of $632.8 \mathrm{~nm}$. Besides, the AuNPs absorb orders of magnitude more photons. The linearity of the signal response for all four modes is not valid beyond a refractive index change of $(\Delta n)$ o.0155, with water as the reference. As predicted in the last section, within a chosen polarization direction, $S_{\text {bulk }}$ value for the first mode $\left(\mathrm{TM}_{1}\right.$ or $\left.\mathrm{TE}_{1}\right)$ is higher than the zeroth mode $\left(\mathrm{TM}_{\mathrm{o}}\right.$ or $\left.\mathrm{TE}_{\mathrm{o}}\right)$. This finding aligns with the established fact that integrated mode intensity increases with mode number because other features of the AuNPs are identical within a defined polarization.

LSPR excited by TM modes are more sensitive than that excited by TE modes. Unlike conventional bulk sensing where the dielectric environment is assumed to be homogeneous throughout plasmonic sensing volume; the bulk sensitivity investigated herein is similar to a conventional short distance sensing in that only the absorption caused by AuNPs very close to the waveguide surface is detected and thus contributes to the waveguide transmission at its end face. Both the waveguide and the AuNPs sense by evanescent fields (although of different nature) with exponentially decaying field intensity. Haes et al. studied the short distance sensitivity of immobilized silver nanostructures by functionalizing them with an alkanethiol and measuring the LSPR shift by standard UV-Vis transmission with unpolarized light at $2 \mathrm{~nm}$ away from the silver surface. ${ }^{25}$ They found that the short distance sensitivity is proportional to out-of-plane height (short axis) and inversely proportional to in-plane length (long axis) of the silver nanostructures. The unpolarized light used in their study is equivalent to the s-polarized light in our study: they both probe along the long axes of plasmonic nanostrucutres that are parallel to the substrate surface. The lower sensitivity of the LSPR induced by TE modes can be attributed to two factors associated with their plasmonic evanescent field: (1) the field intensity decreases due to increased radiative damping effects as the nanoparticle width is increased; (2) the field intensity further decreases due to substrate dielectric effects as larger medium dielectric constants lead to smaller fields. ${ }^{25}$

To the best of our knowledge, no LSPR sensing system based on a multimode slab waveguide and a monochromatic light source has been studied. The most comparable sensitivity experiment was carried out using an optical fiber sensor with AuNPs immobilized electrostatically ${ }^{9}$ or covalently ${ }^{8}$. Their transmission sensitivities normalized

against water were reported to be around one and 4.16 respectively (in RIU ${ }^{-1}$ ). Other groups have reported improved sensitivity of 30 and 35 both in a.u./RIU ${ }^{26-27}$. However, the authors did not comment on their choice of the arbitrary absorption unit, nor did they normalize the sensitivity against a blank sample; therefore, their values are not comparable to the ones obtained above. Other than better structural stability, the ease of mode selection of a multimode slab waveguide is preferable over an optical fiber because sensitivity increases with mode number for a defined polarization. Without any rigorous control in the synthetic techniques of the AuNPs, the highest dipolar sensitivity $\left(\mathrm{TM}_{1}\right.$ mode) of the sensing system is comparable to the quadrupolar sensitivity of a gold nanobipyramid (7.4 RIU1) and higher than its dipolar sensitivity (4.5 RIU $\left.{ }^{-1}\right)$, obtained from wavelength measurement. ${ }^{12}$

Biotin-streptavidin system was used to examine the sensor performance (at $\mathrm{TM}_{1}$ mode) in a biological environment. Thiol solution was prepared in a total concentration of $5 \times 10^{-4} \mathrm{M}$, with 95:5 (molar ratio) of alkane thiol to biotinylated thiol. AuNPs were functionalized with an excess of thiol solution for $12 \mathrm{~h}$. The sensor was exposed to water for a few minutes before the injection of streptavidin to obtain the blank signal. The lowest concentration that leads to a signal increase of more than three times the noise for a blank measurement is $10^{-14} \mathrm{M}$. Indeed, the injection of $10^{-15}$ $\mathrm{M}$ streptavidin solution did not give rise to a detectable 

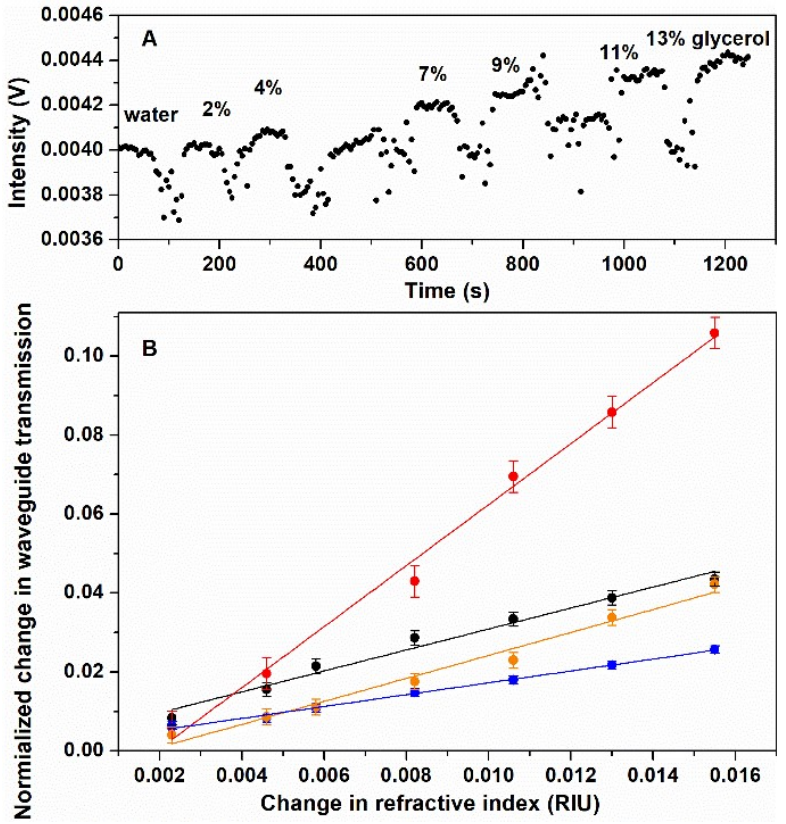

Fig. 5. (A) Real-time transmission intensity of the $\mathrm{TM}_{1}$ mode detected at the waveguide end face. The refractive indices of glycerol solutions: $1.3330,1.3353,1.3376,1.3388,1.3412,1.3436$, $1.3460,1.3485 .{ }^{24}$ The uncertainty in intensity measurement is $\pm 5 \mathrm{X10}^{-6} \mathrm{~V}$. The periodic oscillation of data points is due to the change of liquids and the elimination of air bubbles. (B) Normalized change in waveguide transmission (unitless) with respect to change in refractive index for $\mathrm{TM}_{1}$ (red), $\mathrm{TM}_{\mathrm{o}}$ (black), $\mathrm{TE}_{1}$ (orange), and $\mathrm{TE}_{\mathrm{o}}$ (blue). The $\mathrm{R}^{2}$ values of linear fittings are all above 0.98 .

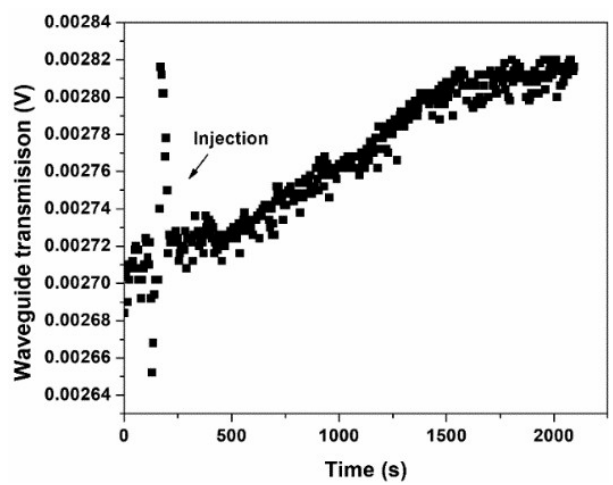

Fig.6. Binding kinetics of streptavidin to biotinylated AuNPs, observed at $\mathrm{TM}_{1}$ mode. The sensor was exposed to water between $\mathrm{o}$ and $220 \mathrm{~s}$. The streptavidin solution was injected around $\mathrm{t}=\mathbf{2 2 0} \mathrm{s}$. The waveguide transmission between 220 and $500 \mathrm{~s}$ is slightly higher than that between o and $220 \mathrm{~s}$ because of an increased bulk refractive index. The blank (water) signal was $2.71 \mathrm{mV}$. The signal increased by $0.083 \mathrm{mV}$ from $2.725 \mathrm{mV}$ to $2.808 \mathrm{mV}$.

signal. Thus, the limit of detection (LOD) for this sensing system is on the order of $10^{-14} \mathrm{M}$, which falls on the lower end of the range for LSPR biosensors., ${ }^{1,28-29}$ Without any optimization, this LOD already approaches that for the most sensitive surface plasmon resonance (SPR) sensor. ${ }^{30}$ If we assume a linear sensor behavior, a normalized signal increase of $0.031(0.083 \mathrm{mV} / 2.71 \mathrm{mV})$ corresponds to a 0.004 increase in refractive index, given that the sensitivity for $\mathrm{TM}_{1}$ mode is $7.744 \mathrm{RIU}^{-1}$. As shown in Fig.6, the kinetic signal increases until all streptavidin molecules are bound to biotin, after which the signal plateaus (from 1500 to 2000 s). The observed signal shape is determined by the diffusion of streptavidin molecules as well as the binding kinetics between biotin and streptavidin. Since the signal increases gradually (from 500 to $1500 \mathrm{~s}$ ) instead of instantaneously as in the bulk sensing experiment, it is not influenced by an increased bulk refractive index introduced by the streptavidin solution.

Factors affecting the sensor performance. We consider three aspects of the sensor performance: sensitivity, stability and reproducibility. The observation wavelength needs to be picked strategically so that the slope of the absorption spectrum is maximized without compromising the reliability, the sensitivity and the linearity of signal response: the steepest section might be too far away from the resonance wavelength, which results in either undetectable signal or signal without a consistent trend. Thanks to the tunability of OMCVD, we have the liberty of testing various AuNP populations with LSPR wavelengths shorter and longer than the observation wavelength (to the shorter and longer wavelength of $632.8 \mathrm{~nm}$ ). When the absorption maximum falls to the left of the observation wavelength (Fig. 7 , red), the observed absorption increases with the redshift of the spectrum, until the LSPR wavelength coincides with the observation wavelength (the red dot to the green dot; a decrease in waveguide transmission). This process is then followed by a further redshift, which leads to a decreased absorption (the green dot to the blue dot) or an increased waveguide transmission. Such inconsistent trend in signal response is undesirable. On the other hand, a sensor with an LSPR wavelength longer than the observation wavelength, such as the system studied here, offers a reliable signal response of increasing waveguide transmission without any reversal (Fig. 2). A certain level of signal fluctuation is expected unless a channel waveguide is implemented; the spreading of light into the z-direction will be not problematic for a micron-wide channel waveguide. Reducing the length of the planar waveguide and increasing the ratio of sensing versus non-sensing area will improve the signal-to-noise ratio, but not significantly.

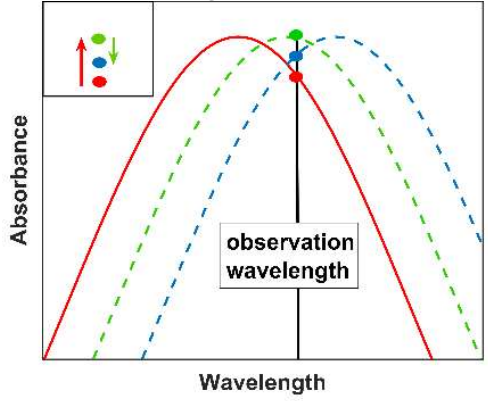

Fig.7. Illustration of the shifting behavior when the initial LSPR wavelength is below the observation wavelength. The initial absorption spectrum (red) exhibits a redshift (green), 
then a further shift (blue). The observed absorption at each stage is highlighted with a dot. The change in absorption with the redshift is indicated with arrows in the insert. The time sequence will be red, green, and blue. Such an inconsistent trend is undesirable.

In terms of mechanical stability, a transition from glass substrates to all-polymer substrates will be implemented in the near future, because the latter is much less fragile and more mass-producible. The coupling grating will be hot embossed from a nickel mold. Although the sensor is designed to function as a disposable device, reproducibility still needs to be considered to ensure a consistent sensor performance. One major drawback of OMCVD is the lack of control over the distribution of nanoparticles, which affects both intensity and wavelength sensing. Our signal is rather less affected by a non-uniform distribution because it is determined by the accumulative absorption of all the AuNPs in the path of a propagating mode; whereas conventional wavelength sensing only measures through a single spot. A bulk sensing calibration can be performed to standardize sensor performance by signal offset. Because the linearity of waveguide transmission with respect to refractive index has been confirmed for all four modes, two points should suffice.

Plasmonic nanoparticles with various shapes and sizes can be covalently immobilized onto the waveguide surface via OMCVD, given the corresponding precursor is available commercially or synthetically. Through an appropriate self-assembled monolayer, the discussed sensing system is able to detect a variety of chemical and biological species. Briefly looking into the future, we expect that with optimization, the sensing system can easily be mass-produced and incorporated into high throughput screening devices.

\section{ASSOCIATED CONTENT}

Supporting Information. Sample Gaussian fitting of the mline peaks for $\mathrm{TM}_{\mathrm{o}}$ and $\mathrm{TM}_{1}$ modes after gold deposition. Parameters for $\mathrm{m}$-line peaks. Average waveguide transmission for each liquid medium. Kinetic measurements for $\mathrm{TE}_{\mathrm{o}}, \mathrm{TM}_{\mathrm{o}}$ and $\mathrm{TM}_{1}$ modes. This material is available free of charge via the Internet at http://pubs.acs.org.

\section{AUTHOR INFORMATION}

\section{Corresponding Author}

* (S.M.). Email: smittler@uwo.ca.

\section{ACKNOWLEDGMENT}

Z.D. and S.M. are grateful to the Natural Sciences and Engineering Research Council (NSERC) for fund RGPIN 201405793. The authors would like to thank Uwe Langbein and Frederik Fleißner (RheinMain University of Applied Sciences, Wiesbaden, Standort Rüsselsheim, Germany) for the fabrication of the grating-substrates. The Western Nanofabrication Facility is thanked for the availability of the UV Ozone generator. The authors would also like to thank Dr. Todd Simpson for taking the electron microscopy images.

\section{REFERENCES}

1. Haes, A. J.; Van Duyne, R. P., A Nanoscale Optical Biosensor: Sensitivity and Selectivity of an Approach Based on the Localized Surface Plasmon Resonance Spectroscopy of Triangular Silver Nanoparticles. J. Am. Chem. Soc. 2002, 124 (35), 10596-10604.

2. Mayer, K. M.; Hafner, J. H., Localized Surface Plasmon Resonance Sensors. Chem. Rev. 2011, 111 (6), 3828-3857.

3. Hutter, E.; Fendler, J. H., Exploitation of Localized Surface Plasmon Resonance. Adv. Mater. 2004, 16 (19), 1685-1706.

4. Kajiura, M.; Nakanishi, T.; Iida, H.; Takada, H.; Osaka, T., Biosensing by optical waveguide spectroscopy based on localized surface plasmon resonance of gold nanoparticles used as a probe or as a label. J. Colloid Interface Sci. 2009, 335 (1), 140-145.

5. Kazuma, E.; Tatsuma, T., Localized surface plasmon resonance sensors based on wavelength-tunable spectral dips. $\mathrm{Na}$ noscale 2014, 6 (4), 2397-2405.

6. Mittler, S., Gold Nanoparticles on Waveguides For and Toward Sensing Application. In Optical Guided-wave Chemical and Biosensors I, Zourob, M.; Lakhtakia, A., Eds. Springer Berlin Heidelberg: Berlin, Heidelberg, 2009; pp 209-229.

7. Burke, C. S.; Polerecky, L.; MacCraith, B. D., Design and fabrication of enhanced polymer waveguide platforms for absorption-based optical chemical sensors. Meas. Sci. Technol. 2oo4, 15 (6), 1140.

8. Lin, H.-Y.; Huang, C.-H.; Cheng, G.-L.; Chen, N.-K.; Chui, H.C., Tapered optical fiber sensor based on localized surface plasmon resonance. Opt. Express 2012, 20 (19), 21693-21701.

9. Chau, L.-K.; Lin, Y.-F.; Cheng, S.-F.; Lin, T.-J., Fiber-optic chemical and biochemical probes based on localized surface plasmon resonance. Sens. Actuators B Chem. 2006, 113 (1), 100-105.

10. Kandeepan, S.; Paquette, J. A.; Gilroy, J. B.; Mittler, S., OMCVD Gold Nanoparticles Covalently Attached to Polystyrene for Biosensing Applications. Chem. Vap. Deposition 2015, 21 (10-1112), 275-28o.

11. Lee, T. M.; Mittler-Neher, S.; Neher, D.; Stegeman, G. I.; Roux, C.; Leclerc, M.; Martin, J.; Najafi, S. I., Side-chain dilution effects on the optical properties of poly[3-alkylthiophene]s. Opt. Mater. 1992, 1 (2), 65-70.

12. Yong, Z.; Lei, D. Y.; Lam, C. H.; Wang, Y., Ultrahigh refractive index sensing performance of plasmonic quadrupole resonances in gold nanoparticles. Nanoscale Res. Lett. 2014, 9 (1), 187187.

13. MacManus, L. F.; Walzak, M. J.; McIntyre, N. S., Study of ultraviolet light and ozone surface modification of polypropylene. J. Polym. Sci., Part A: Polym. Chem. 1999, 37 (14), 2489-2501.

14. Puddephatt, R. J., Reactivity and mechanism in the chemical vapour deposition of late transition metals. Polyhedron 1994, 13 (8), 1233-1243.

15. Tamaki, A.; Kochi, J. K., Formation and decomposition of alkyl-gold(I) complexes. J. Organomet. Chem. 1973, 61, 441-450.

16. Muhich, C. L.; Qiu, J.; Holder, A. M.; Wu, Y.-C.; Weimer, A. W.; Wei, W. D.; McElwee-White, L.; Musgrave, C. B., Solvent Control of Surface Plasmon-Mediated Chemical Deposition of Au Nanoparticles from Alkylgold Phosphine Complexes. ACS Appl. Mater. Interfaces 2015, 7 (24), 13384-13394.

17. Kelly, K. L.; Coronado, E.; Zhao, L. L.; Schatz, G. C., The Optical Properties of Metal Nanoparticles: The Influence of Size, Shape, and Dielectric Environment. J. Phys. Chem. B 2003, 107 (3), 668-677.

18. Jensen, T.; Kelly, L.; Lazarides, A.; Schatz, G. C., Electrodynamics of Noble Metal Nanoparticles and Nanoparticle Clusters. J. Cluster Sci. 1999, 10 (2), 295-317.

19. Aliganga, A. K. A.; Lieberwirth, I.; Glasser, G.; Duwez, A.-S.; Sun, Y.; Mittler, S., Fabrication of equally oriented pancake 
shaped gold nanoparticles by SAM-templated OMCVD and their optical response. Org. Electron. 2007, 8 (2), 161-174.

20. Rafsanjani, S. M. H.; Cheng, T.; Mittler, S.; Rangan, C., Theoretical proposal for a biosensing approach based on a linear array of immobilized gold nanoparticles. J. Appl. Phys. 2010, 107 (9), 094303.

21. Höök, F.; Vörös, J.; Rodahl, M.; Kurrat, R.; Böni, P.; Ramsden, J. J.; Textor, M.; Spencer, N. D.; Tengvall, P.; Gold, J.; Kasemo, B., A comparative study of protein adsorption on titanium oxide surfaces using in situ ellipsometry, optical waveguide lightmode spectroscopy, and quartz crystal microbalance/dissipation. Colloids Surf. B Biointerfaces 2002, 24 (2), 155-170.

22. Marcuse, D. Quantum Electronics-Principles and Applications, 2nd ed.; Academic Press: 1991.

23. Thoma, F.; Langbein, U.; Mittler-Neher, S., Waveguide scattering microscopy. Opt. Commun. 1997, 134 (1), 16-20.

24. Hoyt, L. F., New Table of the Refractive Index of Pure Glycerol at $20^{\circ} \mathrm{C}$. J. Ind. Eng. Chem. 1934, 26 (3), 329-332.

25. Haes, A. J.; Zou, S.; Schatz, G. C.; Van Duyne, R. P., Nanoscale Optical Biosensor: Short Range Distance Dependence of the Localized Surface Plasmon Resonance of Noble Metal Nanoparticles. J. Phys. Chem. B 2004, 108 (22), 6961-6968.

26. Sai, V. V. R.; Kundu, T.; Mukherji, S., Novel U-bent fiber optic probe for localized surface plasmon resonance based biosensor. Biosens. Bioelectron. 2009, 24 (9), 2804-2809.

27. Mitsui, K.; Handa, Y.; Kajikawa, K., Optical fiber affinity biosensor based on localized surface plasmon resonance. Appl. Phys. Lett. 2004, 85 (18), 4231-4233.

28. Fan, M.; Thompson, M.; Andrade, M. L.; Brolo, A. G., Silver Nanoparticles on a Plastic Platform for Localized Surface Plasmon Resonance Biosensing. Anal. Chem. 2010, 82 (15), 6350-6352.

29. Haes, A. J.; Duyne, R. P. V., Preliminary studies and potential applications of localized surface plasmon resonance spectroscopy in medical diagnostics. Expert Rev. Mol. Diagn. 2004, 4 (4), 527-537.

30. Zhang, Q.; Jing, L.; Zhang, J.; Ren, Y.; Wang, Y.; Wang, Y.; Wei, T.; Liedberg, B., Surface plasmon resonance sensor for femtomolar detection of testosterone with water-compatible macroporous molecularly imprinted film. Anal. Biochem. 2014, 463 (Supplement C), 7-14. 